\title{
Fundal intramural ectopic pregnancy
}

\author{
Michael Chaikof MD, Sebastian Hobson MD MPH PhD, Mara Sobel MD MSc
}

Cite as: CMAJ 2020 October 13;192:E1211. doi: 10.1503/cmaj.200181

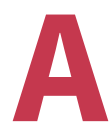

35-year-old woman (G2P2) presented to her physician with abdominal bloating and discomfort, and a positive home pregnancy test. Five years earlier, the patient had a massive postpartum hemorrhage after delivering her second child that required dilatation and curettage. Severe Asherman syndrome (intrauterine adhesions) subsequently developed with complete amenorrhea. This diagnosis was confirmed by diagnostic hysteroscopy, which showed an obliterated endometrial cavity in the presence of synechiae.

Urgent pelvic ultrasonography at the time of this presentation showed a live intramural ectopic pregnancy (estimated gestational age of $8 \mathrm{wk}$ ) located at the uterine fundus. The gestational sac was located within $3 \mathrm{~mm}$ of the uterine serosa, which is associated with a high risk of uterine rupture (Figure $1 \mathrm{~A}$ ).

Owing to the presence of a fetal heartbeat and the pregnancy location, we performed uterine-conserving surgery with fundal hysterotomy and removal of the ectopic pregnancy. We sutured the resultant myometrial defect in multiple layers (Figure 1B). Our patient went home in stable condition on postoperative day 2 .

Ectopic pregnancies complicate $1 \%-2 \%$ of all pregnancies and are a leading cause of maternal mortality worldwide, responsible for a similar number of maternal deaths as preeclampsia and eclampsia. ${ }^{1,2}$ They typically implant in the fallopian tube but can be diagnosed in other locations. ${ }^{1}$ Intramural ectopic pregnancies, which implant in the muscular layer of the uterus, are one of the rarest forms and account for less than $1 \%$ of all ectopic pregnancies ${ }^{3}$. More than $50 \%$ of patients with an intramural pregnancy have had a previous dilatation and curettage. ${ }^{3}$ Uterine surgeries can leave decidual or myometrial defects, which are thought to be a risk factor.

\section{References}

1. Molinaro TA, Barnhart KT. Ectopic pregnancies in unusual locations. Semin Reprod Med 2007;25:123-30.

2. Montgomery A, Hannon G, Muhammad S, et al. Tubal ectopic pregnancies: risk, diagnosis and management. Br J Midwifery 2017;25. doi: 10.12968/ bjom.2017.25.11.700.

3. Kirk E, McDonald K, Rees J, et al. Intramural ectopic pregnancy: a case and review of the literature. Eur J Obstet Gynecol Reprod Biol 2013;168:129-33.

\section{Competing interests: None declared.}

This article has been peer reviewed.

The authors have obtained patient consent.

Affiliations: Department of Obstetrics and Gynecology (Chaikof, Hobson, Sobel), University of Toronto; Department of Obstetrics and Gynecology (Hobson, Sobel), Mount Sinai Hospital, Toronto, Ont.

Correspondence to: Michael Chaikof, michael.chaikof@mail.utoronto.ca 